\title{
Dual-Gated Motion-Frozen Cardiac PET with Flurpiridaz F 18
}

\author{
Piotr J. Slomka ${ }^{1,2}$, Mathieu Rubeaux ${ }^{1}$, Ludovic Le Meunier ${ }^{2}$, Damini Dey ${ }^{1}$, Joel L. Lazewatsky ${ }^{3}$, Tinsu Pan ${ }^{4}$, \\ Marc R. Dweck ${ }^{5}$, David E. Newby ${ }^{5}$, Guido Germano ${ }^{1}$, and Daniel S. Berman ${ }^{1}$ \\ ${ }^{1}$ Departments of Imaging and Medicine, Cedars-Sinai Medical Center, Los Angeles, California; ${ }^{2}$ Siemens Healthcare Molecular \\ Imaging, Knoxville, Tennessee; ${ }^{3}$ Lantheus Medical Imaging, North Billerica, Massachusetts; ${ }^{4}$ MD Anderson Center, Houston, Texas; \\ and ${ }^{5}$ University of Edinburgh, Edinburgh, United Kingdom
}

\begin{abstract}
A novel PET radiotracer, Flurpiridaz F 18, has undergone phase II clinical trial evaluation as a high-resolution PET cardiac perfusion imaging agent. In a subgroup of patients imaged with this agent, we assessed the feasibility and benefit of simultaneous correction of respiratory and cardiac motion. Methods: In 16 patients, PET imaging was performed on a 4-ring scanner in dual cardiac and respiratory gating mode. Four sets of data were reconstructed with high-definition reconstruction (HD·PET): ungated and 8-bin electrocardiographygated images using 5-min acquisition, optimal respiratory gating (ORG) — as developed for oncologic imaging —using a narrow range of breathing amplitude around end-expiration level with $35 \%$ of the counts in a 7-min acquisition, and 4-bin respiration-gated and 8-bin electrocardiography-gated images (32 bins in total) using the 7-min acquisition (dual-gating, using all data). Motion-frozen (MF) registration algorithms were applied to electrocardiography-gated and dual-gated data, creating cardiac-MF and dual-MF images. We computed wall thickness, wall/cavity contrast, and contrast-to-noise ratio for standard, ORG, cardiac-MF, and dual-MF images to assess image quality. Results: The wall/cavity contrast was similar for ungated $(9.3 \pm 2.9)$ and ORG (9.5 \pm 3.2$)$ images and improved for cardiac-MF (10.8 \pm 3.6$)$ and dual-MF images $(14.8 \pm 8.0)(P<0.05)$. The contrast-to-noise ratio was $22.2 \pm 9.1$ with ungated, $24.7 \pm 12.2$ with $O R G, 35.5 \pm 12.8$ with cardiac-MF, and $42.1 \pm 13.2$ with dual-MF images (all $P<0.05$ ). The wall thickness was significantly decreased $(P<0.05)$ with dualMF $(11.6 \pm 1.9 \mathrm{~mm})$ compared with ungated $(13.9 \pm 2.8 \mathrm{~mm})$, ORG $(13.1 \pm 2.9 \mathrm{~mm})$, and cardiac-MF images $(12.1 \pm 2.7 \mathrm{~mm})$. Conclusion: Dual (respiratory/cardiac)-gated perfusion imaging with Flurpiridaz F 18 is feasible and improves image resolution, contrast, and contrastto-noise ratio when MF registration methods are applied.
\end{abstract}

Key Words: cardiology (basic/technical); cardiology (clinical); PET; cardiac PET; flurpiridaz; motion-frozen

J Nucl Med 2015; 56:1876-1881

DOI: 10.2967/jnumed.115.164285

$\mathbf{T}$ he image quality of myocardial perfusion images acquired with a PET system depends on the radiotracer used in the study. The

Received Jul. 21, 2015; revision accepted Sep. 14, 2015.

For correspondence or reprints contact: Piotr J. Slomka, Artificial Intelligence in Medicine Program, 8700 Beverly Blvd., Suite A047N, Los Angeles, CA 90048.

E-mail: piotr.slomka@cshs.org

Published online Sep. 24, 2015.

COPYRIGHT (C) 2015 by the Society of Nuclear Medicine and Molecular Imaging, Inc. commonly used radiotracers in cardiac PET imaging are ${ }^{15} \mathrm{O}$-water $\left(\mathrm{H}_{2}{ }^{15} \mathrm{O}\right)$, ammonia $\left({ }^{13} \mathrm{NH}_{3}\right)$, and 82-rubidium (1). A new ${ }^{18} \mathrm{~F}$ based radiotracer for PET myocardial perfusion imaging (Flurpiridaz F 18; Lantheus Medical Imaging) demonstrated excellent image quality in a phase I clinical trial (2) and better image quality, interpretative certainty, and diagnostic performance than SPECT in a phase 2 clinical trial (3). In particular, imaging with this ${ }^{18} \mathrm{~F}$-based radiotracer generated higher-resolution cardiac images (4).

However, the high image resolution associated with an ${ }^{18} \mathrm{~F}-$ based tracer can be degraded by cardiac and respiratory motion during PET acquisition, leading to image blurring. If such motion is not corrected, the full-potential imaging resolution of Flurpiridaz F 18 may not be realized. We have previously developed a motion-frozen (MF) technique for myocardial perfusion imaging, which recovers resolution lost due to cardiac motion, improving contrast and quantitative diagnostic accuracy $(5,6)$. This technique was also successfully used for automatic coregistration of SPECT with CT angiography (CTA) $(7,8)$ and for contrast improvement in cardiac PET (9).

The aim of this study was to investigate the feasibility of myocardial perfusion imaging by Flurpiridaz F 18 PET with correction of both cardiac and respiratory motion. We hypothesized that the myocardial perfusion image quality for Flurpiridaz F 18 would be improved with dual-gated MF techniques beyond what is possible with the resolution recovery during reconstruction and MF techniques, which correct for cardiac motion only.

\section{MATERIALS AND METHODS}

\section{Patients}

The study population consisted of 16 patients (12 men and 4 women) recruited into a phase II clinical trial of Flurpiridaz F 18. The inclusion and exclusion criteria are given in the report of the clinical trial (3). Patients were instructed to discontinue caffeinated drinks and nitrate medications for $24 \mathrm{~h}$ and $\beta$-blockers and calcium antagonists for $48 \mathrm{~h}$ before testing. The study was approved by the institutional review board, and all patients provided written informed consent.

\section{PET Acquisition}

All patients underwent a rest-stress Flurpiridaz F 18 PET/CT scan as previously described (3). All images were acquired on a Siemens Biograph64 TruePoint PET/CT scanner with the TrueV option. This 3-dimensional system consists of a 64-slice CT and a PET scanner with 4 rings of lutetium oxyorthosilicate detectors with a detector element dimension of $4 \times 4 \times 20 \mathrm{~mm}(10)$. The image plane spacing was $2 \mathrm{~mm}$. The PET axial and transaxial fields of view were 216 and $605 \mathrm{~mm}$, respectively. The coincidence time window and the energy window were $4.5 \mathrm{~ns}$ and $425-$ $650 \mathrm{keV}$, respectively. The data were acquired in list-mode format. A full description of the system performance has been previously described (11). 


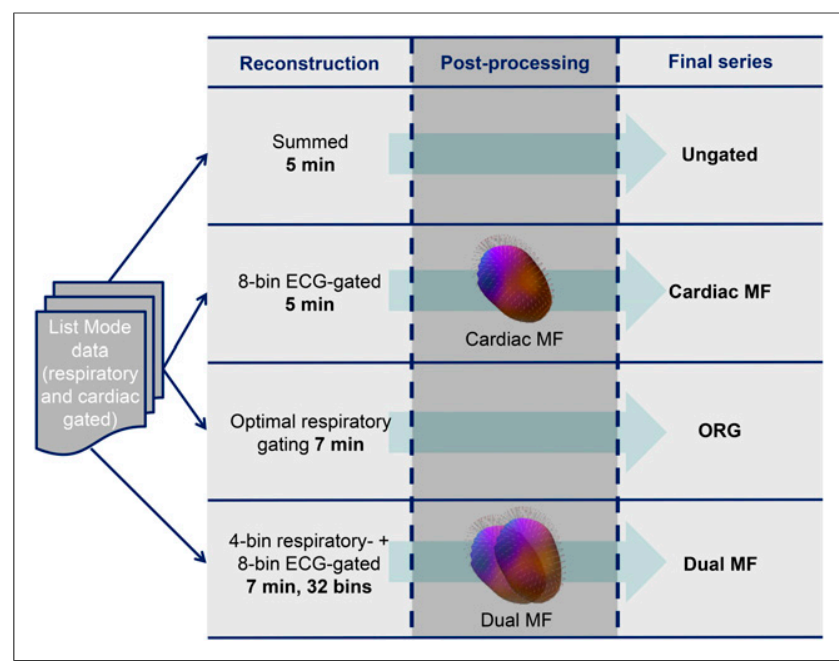

FIGURE 1. Overview of reconstructed datasets. MF = motion frozen; $\mathrm{ORG}=$ optimal respiratory gating; dual $\mathrm{MF}=$ cardiac and respiratory MF processing. After postprocessing, all final series are static.

After a 2.8-s standard topogram acquisition $(120 \mathrm{kVp})$, patients underwent a CT scan for attenuation correction (CTAC) using the following parameters: spiral mode; slice thickness, $3.0 \mathrm{~mm}$; total scan time, $3.36 \mathrm{~s}$; pitch, 1.5 ; rotation time, $0.5 \mathrm{~s}$; collimation, $24 \times 1.2 \mathrm{~mm}$; tube voltage, $120 \mathrm{kVp}$; and tube current, $11 \mathrm{mAs}$. The estimated radiation dose for this CTAC acquisition was $0.3 \mathrm{mSv}$. During the CTAC scan, the patients were instructed to perform normal end-expiration breath-hold. Rest perfusion images were acquired for 1 bed position around the heart (identical to the CT coverage of $210 \mathrm{~mm}$ ) immediately on infusion of $85.1-159.1 \mathrm{MBq}$ (2.3-4.3 mCi) of Flurpiridaz F 18 (depending on stressing protocol). Images were acquired for $15 \mathrm{~min}$. Stress testing was performed either with a symptom-limited Bruce treadmill exercise protocol or with intravenous adenosine $(140 \mu \mathrm{g} / \mathrm{kg} / \mathrm{min}$ for $6 \mathrm{~min})$. At near-maximal exercise, 277.5-388.5 MBq (7.5-10.5 mCi) of Flurpiridaz F 18 (depending on stressing protocol) were injected intravenously, after which treadmill exercise was continued at maximal workload for $1 \mathrm{~min}$ and at 1 stage lower for 2 additional minutes whenever possible. The 15-min perfusion PET acquisition was started at least $25 \mathrm{~min}$ after termination of exercise. For pharmaceutical stress with adenosine, Flurpiridaz F 18 was injected at the end of the third minute and a 15-min PET acquisition was started immediately. The interval between the rest and stress acquisitions was either $30 \mathrm{~min}$ when the patient was stressed with adenosine or $60 \mathrm{~min}$ when exercised stress was used. Twelve-lead electrocardiography was monitored continuously during stress testing.

The rest studies were acquired in cardiac-gating mode only whereas dual-gating (cardiac and respiratory) was used for all stress studies to minimize interference with the clinical trial. Therefore, we considered only stress studies here. The cardiac gating was performed with a 3-lead electrocardiography gating system inside the PET gantry. Respiratory gating was performed using a prototype pneumatic belt (Ivy Biomedical Inc.). This device sends the amplitude of the breathing motion to the PET system every millisecond. We used a modified acquisition control system to code the amplitude as tag-words inside the list-mode file at appropriate time positions. The amplitude-based respiratory gating has been shown to be more effective than the phase-based gating (12) with patients showing an irregular breathing pattern.

\section{Reconstruction Protocols}

List-mode files with embedded dual-gating signals were processed using an in-house-developed rebinning application. The extraction of the respiratory and cardiac signals allowed limitation of the reconstruction to specific sections of the cardiac or respiratory cycle. All images were reconstructed after a 5-min delay to allow for blood-pool clearance.

Rebinning. Two different reconstruction protocols were used for respiratory gating. The first one sorted the PET data list with an amplitude-based respiratory gating method, designed to keep the $35 \%$ of the PET counts in 1 optimal respiratory gate (ORG), in which the breathing motion was minimal (13).

In the second rebinning method, 4 separate list-mode files corresponding to 4 different breathing amplitudes were generated. We kept $20 \%$ of the total data in each of the 4 list-mode files corresponding to each respiratory gate, leaving out approximately $20 \%$ of events corresponding to the most extreme expiration and inspiration motion. The resulting 4 bins had equal time duration (but variable amplitude), which had the advantage of providing the same statistics in each bin. This initial rebinning of list-mode data allowed us to reconstruct dual-gated datasets.

Static and 8-bin electrocardiography-gated sinograms were then generated from all list-mode files. The sinograms from the original acquisition data list (no respiratory gating) included $5 \mathrm{~min}$ of the acquisition whereas the respiratory-gated and dual-gated sinograms were generated using 7 min of the acquisition to compensate for the reduced number of counts due to the respiratory rebinning, both starting 5 min after Flurpiridaz F 18 injection to allow for blood flow clearance.

\section{Reconstruction}

After the data list file sinograms were sorted, static summed images and 8-bin electrocardiography-gated images were reconstructed with HD • PET method (4 iterations and 14 subsets), which takes advantage of resolution recovery principles $(14,15)$. For the fully dual-gated sinograms, 8-bin electrocardiography-gated by 4-bin respiratory-gated images were generated with HD - PET for a total of 32 bins. The reconstructed fully dual-gated datasets can be processed as 4 separate 8-bin electrocardiography-gated datasets, each of them at a different respiratory level. Figure 1 summarizes all the different reconstructions evaluated in this study. We chose to filter all patients with a 2-mm gaussian filter to maintain the same resolution and minimize noise suppression. The reconstruction matrix was $168 \times 168 \times 109$ with a zoom of 2 , and the pixel size was $2 \times 2 \times 2 \mathrm{~mm}$. Scatter, decay, and random corrections were applied to the reconstructed images. Standard CT-based attenuation correction was used. When a misalignment was identified, a manual registration matrix with 3-dimensional translations was generated by an experienced technologist and applied before the final reconstruction process.

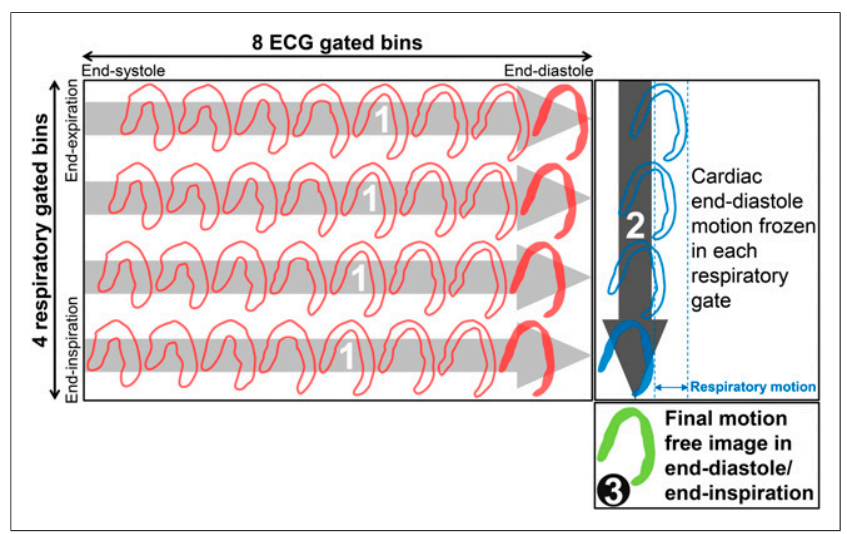

FIGURE 2. Illustration of dual-respiratory/cardiac-MF technique: 1. Cardiac-MF processing separately in each respiratory phase (contoured red bins) to end-diastole phase (solid red bins). 2. Respiratory MF processing of cardiac end-diastole bins (contoured blue bins) to end-inspiration bin (solid blue bin). 3. Final motion-free image in enddiastole/end-inspiration (solid green bin). ECG = electrocardiography. 


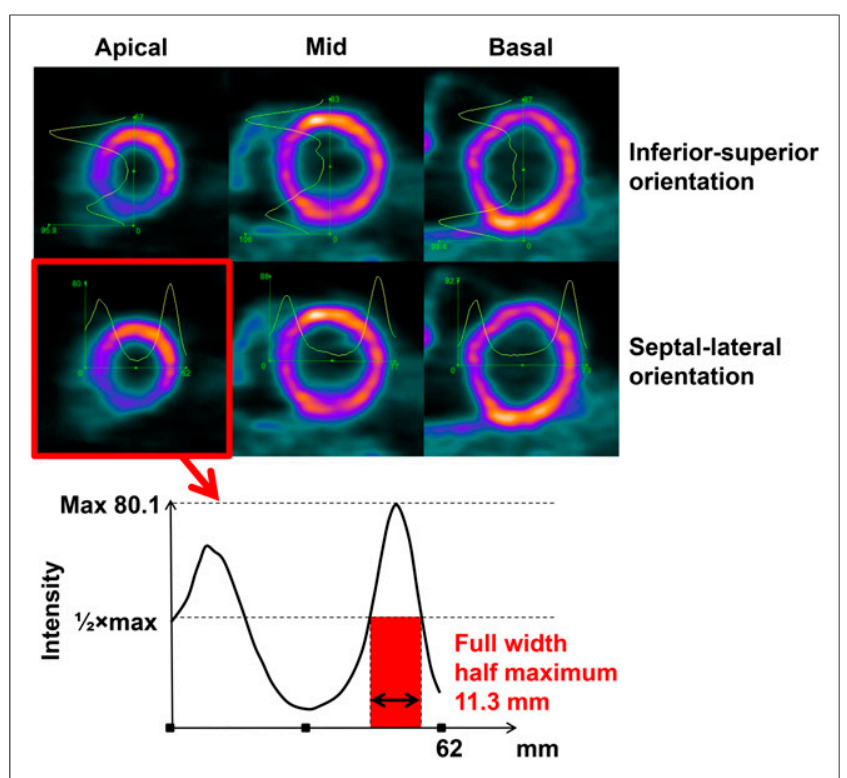

FIGURE 3. Illustration of wall thickness calculation. Wall thickness is defined as the full width half maximum of a profile taken on images at 3 different levels of long axis (basal, mid, and apical) and with 2 orientations (septal-lateral and inferior-superior) for a total of 6 profiles per dataset. Max $=$ maximum

Both the original PET/CT alignment and the alignment after manual registration were checked by an expert imaging physician. For the dual-gated datasets, 4 different PET/CT registration matrices were generated: 1 for each respiratory phase. Manual registration between PET and CT was applied before reconstruction when a misalignment was identified.

\section{Postprocessing Applied to Reconstructed Image}

MF Technique. MF processing tracks the motion of the left ventricle (LV) in cardiac-gated images and then adjusts all cardiac phases to enddiastolic position, resulting in an image free of cardiac motion. Motion freezing is accomplished by motion tracking of the detected LV endoand epicardial borders $(5,9)$. The algorithm generates 3-dimensional displacement vectors between each cardiac phase and uses those vectors to shift all the counts to the end-diastolic phase. The result is an image free of cardiac motion blurring (i.e., with the same spatial resolution as the single cardiac phase image) but with greater counts and similar noise as compared with static summed images. This algorithm has been previously described in detail and validated for SPECT versus invasive angiography (5).

Dual-MF Technique. The dual-MF technique was applied in 2 consecutive steps. Starting from the 4 separate 8-bin cardiac-gated sets (each corresponding to a different respiratory phase), the MF technique previously described was first applied to shift 8 cardiac bins to end-diastolic position, separately for each respiratory bin. This resulted in 4 cardiac motion-corrected respiratory bins, each adjusted to a separate end-diastolic position. Subsequently, to compensate for the respiratory motion, we applied rigid transformation (6 parameters including 3 translations and 3 rotations), accounting for the difference between reference and target frames by performing consecutive registrations. For the reference frame, we selected the first frame, representing the position during end-expiration. We applied a bilateral filter (16) to remove noise while preserving edge information and generated a binary mask to localize the region of interest (myocardium). Consequently, automated frame-toframe rigid registration of frames $2-4$ to frame 1 (end-expiration) was performed using a similarity measure of normalized cross correlation and gradient descent minimization, in which sample voxels are selected from the mask region for computational efficiency. This algorithm was previously developed by our group for gross patient motion correction of PET data (17). The overview of the dual-respiratory/cardiac-MF processing is shown in Figure 2.

\section{Image Processing and Analysis}

Short-axis reorientation and automatic contouring of the LV in static and gated images were done automatically using QPET software (Cedars Sinai cardiac package) for which the inputs were the transverse slices of the reconstructed PET image (18). MF and dual-MF image processing techniques were then applied to the gated datasets (Fig. 1). The myocardial wall segment analysis was performed using the 17-segment American Heart Association model (19).

Myocardial Wall Thickness. Using the LV coordinates automatically detected by QPET, we estimated the wall thickness defined as the full width half maximum of a profile taken on the images at 3 different levels of the long axis (basal, mid, and apical) and with 2 orientations (septal-lateral and inferior-superior) for a total of 6 profiles per dataset (Fig. 3).

Myocardium-to-Blood Contrast and Contrast-to-Noise Ratio (CNR). Volumes of interest over the LV and the blood pool (inside the LV cavity) were automatically derived on the basis of the QPET segmentation of the LV. Their dimensions were significantly larger than the spatial resolution of the images. We then calculated the contrast between the blood pool and the myocardial wall, and the CNR as follows:

$$
\begin{gathered}
\text { Contrast }=\frac{\text { Mean }(\mathrm{LV})}{\text { Mean (blood) }} \\
\mathrm{CNR}=\frac{\text { Mean }(\mathrm{LV})-\text { Mean (blood) }}{\mathrm{SD}(\text { blood })},
\end{gathered}
$$

where SD is the SD of the blood pool. Contrast and CNR were calculated globally and for each of the 17 segments as defined by American Heart Association, from static summed images and MF processed images.

\section{Statistical Analysis}

All continuous variables are expressed as mean \pm SD. Paired $t$ tests were used to compare differences in paired continuous data, and the McNemar test was used to compare differences in paired discrete data. For unpaired continuous data, 1-way ANOVA was used. All statistical tests were 2 -tailed, and a $P$ value of less than 0.05 was considered significant.

TABLE 1

Patient Demographic and Clinical Characteristics $(n=16)$

\begin{tabular}{lc}
\hline \multicolumn{1}{c}{ Characteristic } & Mean \pm SD or $n(\%)$ \\
\hline Mean age \pm SD $(\mathrm{y})$ & $56 \pm 14$ \\
\hline Men $(\%)$ & $12(75 \%)$ \\
\hline Mean weight $\pm \mathrm{SD}(\mathrm{kg})$ & $83.5 \pm 12.7$ \\
\hline Mean body mass index $\left(\mathrm{kg} / \mathrm{m}^{2}\right) \pm \mathrm{SD}$ & $28 \pm 4$ \\
\hline Normal flurpiridaz $(\%)$ & $6(37.5 \%)$ \\
\hline Smokers $(\%)$ & $4(25 \%)$ \\
\hline Adenosine or exercise, $\mathrm{n}$ adenosine $(\%)$ & $4(25 \%)$ \\
\hline Mean ejection fraction $\pm \mathrm{SD}$ & $62 \pm 9$ \\
\hline
\end{tabular}


TABLE 2

Myocardial Wall Thickness (in mm) Measured on Profiles Taken at Basal, Mid, and Apical Levels in Septal-Lateral and in Superior-Inferior Directions

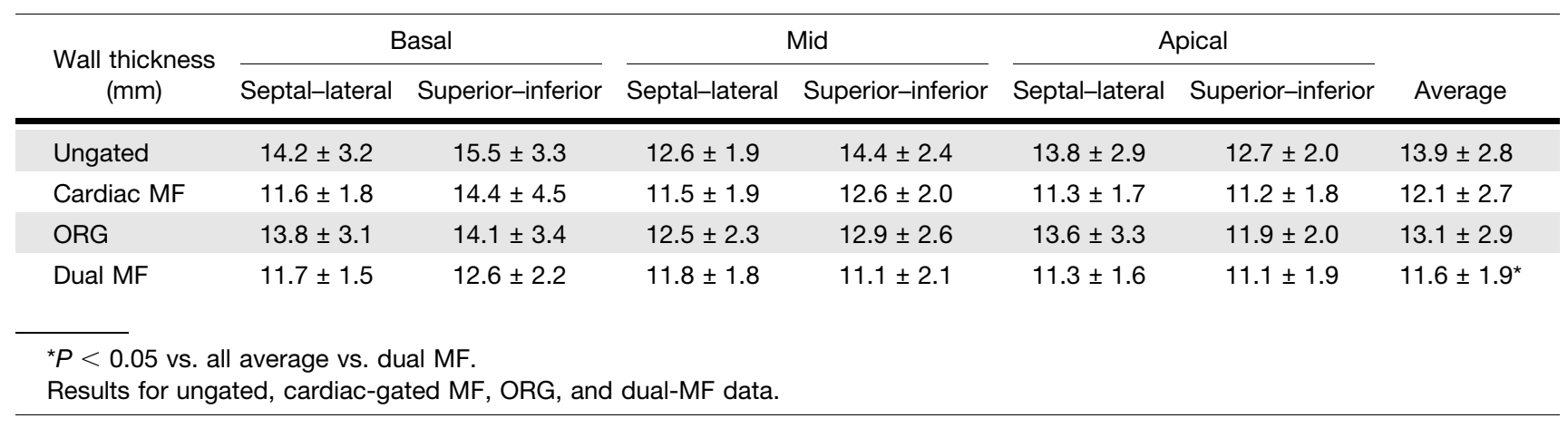

\section{RESULTS}

Patient demographic characteristics are shown in Table 1.

\section{Myocardial Wall Thickness}

The measured wall thickness was significantly different between all datasets. We found that the wall thickness was $13.9 \pm 2.8 \mathrm{~mm}$ for ungated, $12.1 \pm 2.7 \mathrm{~mm}$ for cardiac-MF, $13.1 \pm 2.9 \mathrm{~mm}$ for ORG, and $11.6 \pm 1.9 \mathrm{~mm}$ for dual-MF images (Table 2). The dualMF technique resulted in a decreased wall thickness for $94 \%, 77 \%$, and $91 \%$ of the measurements as compared with ungated, cardiacMF, and ORG data, respectively. For comparison, we also measured the wall thickness for the end-diastole end-expiration phase of the dual-gated datasets, which was $10.7 \pm 2.3 \mathrm{~mm}$.

\section{Myocardium-to-Blood Contrast and CNR}

The myocardium-to-blood contrast significantly changed between the different gating and processing techniques as shown in Figure 4. Figure 5 provides CNR results on a per-patient/per-method basis. Examples of improvements in terms of visual image quality with the different gating and processing techniques can be seen in Figures 6 and 7.

\section{DISCUSSION}

We have shown that with postprocessing techniques, dual-gating is possible with Flurpiridaz F 18 myocardial perfusion imaging and that this approach provides almost motion-free images with low noise and greater apparent resolution. These techniques therefore hold major promise to improve the sensitivity and resolution of myocardial perfusion imaging studies and cardiac PET imaging in general.

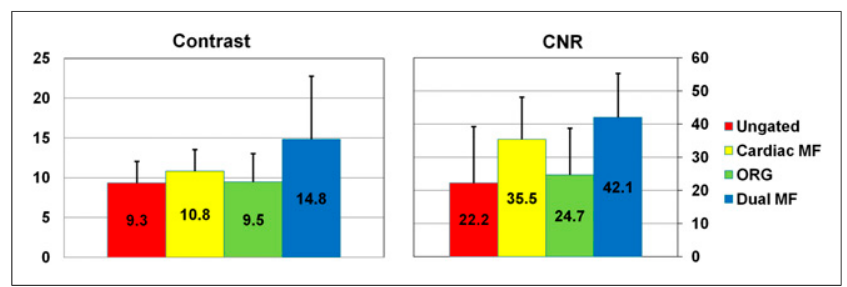

FIGURE 4. Myocardial-to-blood contrast and CNR for ungated, cardiac MF with no respiratory gating, ORG, and dual (respiratory and cardiac)-MF images. $P<0.05$ for all except ungated vs. ORG.
We corrected for both cardiac and respiratory motion that would usually blur the typical static summed perfusion images. We first investigated the feasibility of decreasing the effect of the respiratory motion with an optimal respiratory gating technique that keeps only a narrow range of breathing amplitude $(35 \%)$ around the endexpiration. van Elmpt et al. have previously applied this approach in PET-based radiation therapy planning, though they used different methods to measure and record respiration (13). Another recent publication also considered the same approach (20). This technique eliminates about $65 \%$ of the counts and uses $35 \%$ of the remaining counts in the quiescent phase of the breathing to provide images almost free of respiratory motion.

Although in the original Lantheus protocol, $15 \mathrm{~min}$ of PET emission data (10 min acquisition starting $5 \mathrm{~min}$ after injection to allow clearance from blood pool) were collected to allow for the range of scanners used in the trial, our center had established that a 5 min perfusion acquisition provided clinically robust image quality on the 4-ring Siemens Biograph PET/CT scanner. We increased the acquisition time from 5 to $7 \mathrm{~min}$ for respiratory- and dual-gated datasets to allow similar contrast-to-noise levels for all data. Further, a shorter imaging time has the added benefit of reducing the potential for patient motion, which could confound the effects of respiratory and cardiac motion.

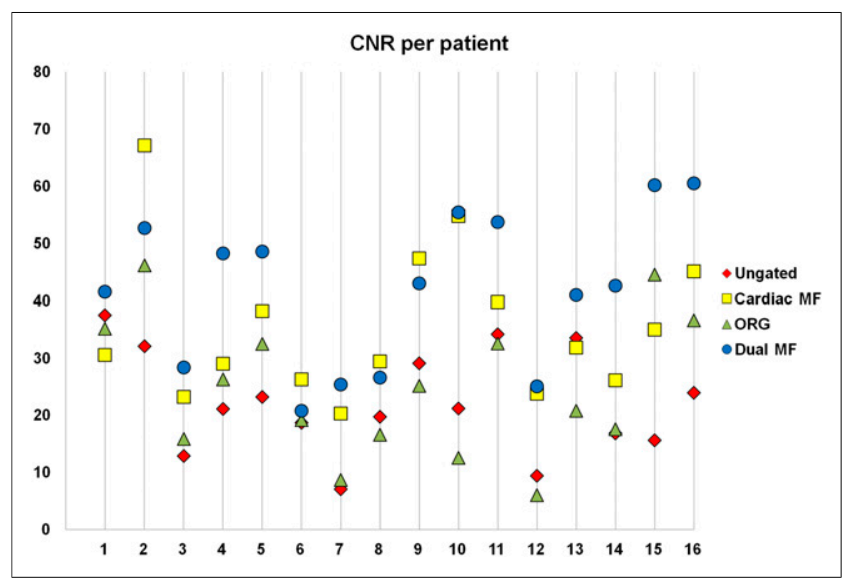

FIGURE 5. CNR for ungated, cardiac MF with no respiratory gating, ORG, and dual (respiratory and cardiac)-MF images, for each of the 16 patients. 


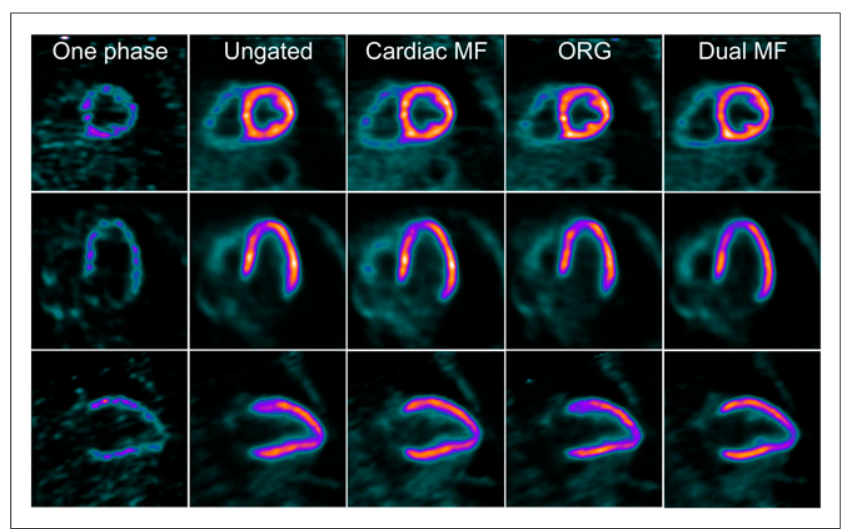

FIGURE 6. A 42-y-old female patient with low likelihood of coronary artery disease (weight, $86.2 \mathrm{~kg}$ [190 lbs]; body mass index, 34.7) injected with $284.9 \mathrm{MBq}(7.7 \mathrm{mCi})$ of Flurpiridaz $\mathrm{F} 18$ during exercise stress and acquired in dual (cardiac and respiratory) mode. Short-, vertical-, and horizontal-axis views of adenosine stress images. 1 phase $=1$ cardiac gate in end-inspiration; dual MF = dual (cardiac/ respiratory) MF; MF = cardiac MF no respiratory gating.

Because the ORG method wastes about $65 \%$ of the counts, we looked into the feasibility of full dual-gated imaging where the MF technique is applied for both respiratory and cardiac motion correction. The CNR in the dual-MF images using $7 \mathrm{~min}$ of acquisition was higher than in the ORG images and consequently the increase in acquisition time may not be needed for the dual-MF technique to keep the noise level similar to the static summed perfusion images.

Correcting for both cardiac and respiratory motion leads to a decreased myocardial wall thickness. The wall thickness has decreased by about $17 \%$ for the dual-MF technique as compared with the standard ungated method currently used for perfusion imaging. These results are closer to average thickness as reported by MR imaging techniques $(10.3 \pm 1.2 \mathrm{~mm}$ at end-diastole $)(21)$. The myocardium-to-blood contrast increased more than $59 \%$ and CNR increased more than $89 \%$ for the dual-MF technique as compared with the reference ungated technique. Even when compared with a cardiac-only MF method, the dual-MF method allowed a $37 \%$

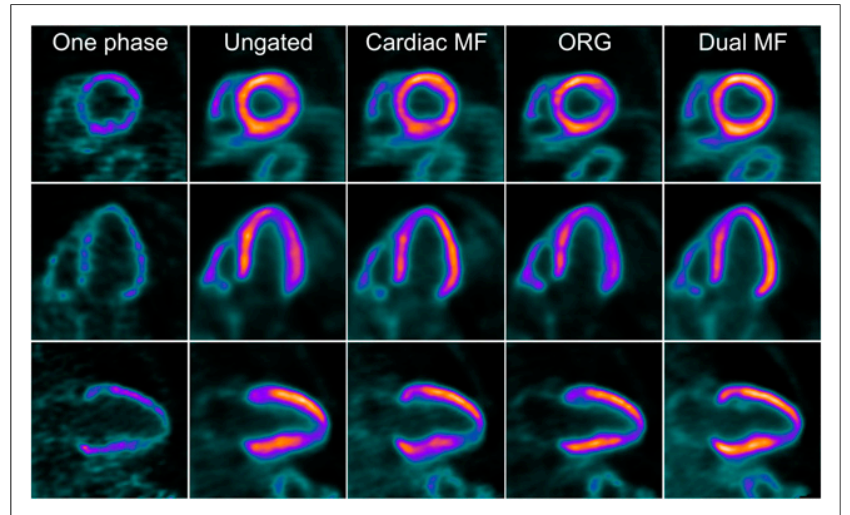

FIGURE 7. A 67-y-old male patient with prior myocardial infarction (weight, $88.5 \mathrm{~kg}$ [195 lbs]; body mass index, 25.7) injected with 355.2 $\mathrm{MBq}(9.6 \mathrm{mCi})$ of Flurpiridaz $\mathrm{F} 18$ during adenosine stress and acquired in dual (cardiac and respiratory) mode. Short-, vertical-, and horizontalaxis views of adenosine stress images. 1 phase $=1$ cardiac gate in endinspiration; dual MF = dual (cardiac/respiratory) MF; MF = cardiac MF no respiratory gating. increase in contrast and 19\% increase in CNR. There were 4 cases, however (Fig. 5), in which the dual-gated technique did not improve CNR over the cardiac-MF method, despite improving contrast. This noise increase is likely due to difficulties in convergence of the iterative reconstruction for low-count individual doublegated bins. More robust reconstruction techniques (22) could be used in future studies to reduce this effect. The ungated images in this study were reconstructed with the state-of-the-art technique incorporating resolution recovery principles, which has been shown to have better quality than regular iterative reconstruction for cardiac PET (15).

This is the first attempt to optimize the reconstruction of myocardial perfusion Flurpiridaz F 18 images with respect to cardiac and respiratory motion. Dual-gating (cardiac/respiratory) (23-26) and preliminary methods for dual motion correction for phantom data and ${ }^{18}$ F-FDG have been reported (27-29) but have not yet been applied to myocardial perfusion imaging and did not use the advanced reconstruction with resolution recovery. Clinical implementation of the dual-gating technique will likely lead to the improved clinical utility of the Flurpiridaz F 18 images but nevertheless adds additional technical complexity. Although the amplitudebased respiratory gating systems are now routinely used for oncologic applications, the registration methods and the 2-step list-mode rebinning used in this study are not yet widely used and may require new streamlined software before they can be routinely applied in clinical practice. Further, in this study we ignored the effect of misalignment of the attenuation scans with individual respiratory and cardiac gates of the PET emission data. Ideally, attenuation maps should be corrected for both cardiac and respiratory motion before the reconstruction of individual bins. However, this will require the use of more complex methods not yet available clinically (30).

Significant improvements in spatial resolution and contrast resolution are possible with an ${ }^{18} \mathrm{~F}$-labeled tracer such as Flurpiridaz F 18, because its positron range is short $(0.54 \mathrm{~mm})$. Therefore, the correction of cardiac and respiratory motion may be of particular importance in this case, allowing the full benefit of improved perfusion image quality for this tracer. Motion as small as $5 \mathrm{~mm}$ can significantly affect the quantitative perfusion analysis, and the analysis of subtle changes between stress and rest myocardial perfusion PET may be affected by changes as small as 5\%-10\% in the reduction of the apparent maximal activity (31). In our previous work applying MF techniques to SPECT, correcting for cardiac motion only, we were able to improve the diagnostic accuracy of the techniques $(5,6)$.

There was no gold standard for wall thickness but end-diastole end-expiration dual-gated phase thickness was measured and compared with dual-MF results. Although apparent contrast and CNR have increased, the clinical significance of these improvements and in particular the potential improvement of perfusion abnormality detection will need to be determined in future work. Our cohort of patients had a limited range of body mass index, typical of our patient population. Our sample size was small $(n=16)$, and further studies with a larger number of patients are warranted to assess more fully the diagnostic value of this technique.

\section{CONCLUSION}

In this work, we showed that dual-gating with cardiac and respiratory motion correction was feasible with Flurpiridaz F 18. Dual-gated MF imaging provides high-quality motion-free perfusion images with improved contrast and image resolution. 


\section{DISCLOSURE}

The costs of publication of this article were defrayed in part by the payment of page charges. Therefore, and solely to indicate this fact, this article is hereby marked "advertisement" in accordance with 18 USC section 1734. Ludovic Le Meunier is an employee of Siemens Medical Systems. Joel L. Lazewatsky is an employee of Lantheus Medical Imaging. Piotr J. Slomka and Daniel S. Berman have received research funding from Siemens. Daniel S. Berman has received research support from Lantheus. No other potential conflict of interest relevant to this article was reported.

\section{ACKNOWLEDGMENT}

We thank Jim Hamill for his help with the optimal respiratory gating method.

\section{REFERENCES}

1. Dilsizian V, Bacharach S, Beanlands R, et al. PET myocardial perfusion and metabolism clinical imaging. J Nucl Cardiol. 2009;16:651-681.

2. Yu M, Guaraldi M, Mistry M, et al. Bms-747158-02: a novel PET myocardial perfusion imaging agent. J Nucl Cardiol. 2007;14:789-798.

3. Berman DS, Maddahi J, Tamarappoo BK, et al. Phase II safety and clinical comparison with single-photon emission computed tomography myocardial perfusion imaging for detection of coronary artery disease: flurpiridaz $\mathrm{F} 18$ positron emission tomography. J Am Coll Cardiol. 2013;61:469-477.

4. Berman DS, Germano G, Slomka PJ. Improvement in PET myocardial perfusion image quality and quantification with flurpiridaz F 18. J Nucl Cardiol. 2012;19 (suppl 1):S38-S45.

5. Slomka PJ, Nishina H, Berman DS, et al. "Motion-frozen" display and quantification of myocardial perfusion. J Nucl Med. 2004;45:1128-1134.

6. Suzuki Y, Slomka PJ, Wolak A, et al. Motion-frozen myocardial perfusion SPECT improves detection of coronary artery disease in obese patients. $J$ Nucl Med. 2008;49:1075-1079.

7. Slomka PJ, Cheng VY, Dey D, et al. Quantitative analysis of myocardial perfusion SPECT anatomically guided by coregistered 64-slice coronary CT angiography. J Nucl Med. 2009;50:1621-1630.

8. Woo J, Slomka PJ, Dey D, et al. Geometric feature-based multimodal image registration of contrast-enhanced cardiac CT with gated myocardial perfusion SPECT. Med Phys. 2009;36:5467-5479.

9. Le Meunier L, Slomka PJ, Dey D, et al. Motion frozen ${ }^{18}$ F-FDG cardiac PET. J Nucl Cardiol. 2011;18:259-266.

10. Jonsson C, Odh R, Schnell PO, Larsson SA. A comparison of the imaging properties of a 3-and 4-ring Biograph PET scanner using a novel extended NEMA phantom. IEEE Nucl Sci Symp Conf Rec. 2007;4:2865-2867.

11. Townsend D, Jakoby B, Long M, et al. Performance and clinical workflow of a new combined PET/CT scanner [abstract]. J Nucl Med. 2007;48(suppl 2):437P.

12. Dawood M, Büther F, Lang N, Schober O, Schäfers KP. Respiratory gating in positron emission tomography: a quantitative comparison of different gating schemes. Med Phys. 2007;34:3067-3076.
13. van Elmpt W, Hamill J, Jones J, De Ruysscher D, Lambin P, Ollers M. Optimal gating compared to 3D and 4D PET reconstruction for characterization of lung tumours. Eur J Nucl Med Mol Imaging. 2011;38:843-855.

14. Kadrmas DJ, Casey M, Conti M, Jakoby B, Lois C, Townsend D. Impact of timeof-flight on PET tumor detection. J Nucl Med. 2009;50:1315.

15. Le Meunier L, Slomka P, Dey D, et al. Enhanced definition PET for cardiac imaging. J Nucl Cardiol. 2010;17:414-426.

16. Tomasi C, Manduchi R. Bilateral filtering for gray and color images. Paper presented at: Sixth IEEE International Conference on Computer Vision; January 1998; Bombay, India.

17. Woo J, Tamarappoo B, Dey D, et al. Automatic 3D registration of dynamic stress and rest $82-\mathrm{Rb}$ and flurpiridaz F 18 myocardial perfusion PET data for patient motion detection and correction. Med Phys. 2011;38:6313-6326.

18. Slomka P, Germano G, Kavanagh PB, Javadi M, Berman DS, Bengel FM. Evaluation of a new automatic algorithm for quantification of ECG-gated $82 \mathrm{Rb}$ cardiac PET [abstract]. J Nucl Med. 2009;50(suppl 2):217P.

19. Cerqueira M, Weissman N, Dilsizian V, et al. Standardized myocardial segmentation and nomenclature for tomographic imaging of the heart: a statement for healthcare professionals from the Cardiac Imaging Committee of the Council on Clinical Cardiology of the American Heart Association. Circulation. 105;4:539542.

20. Liu C, Alessio A, Pierce L, et al. Quiescent period respiratory gating for PET/CT. Med Phys. 2010;37:5037-5043.

21. Kaul S, Wismer G, Brady T, et al. Measurement of normal left heart dimensions using optimally oriented MR images. AJR. 1986;146:75.

22. Hong I, Cho S, Casey M, Michel C. Complementary reconstruction: improving image quality in dynamic PET studies. Paper presented at: IEEE Nuclear Science Symposium and Medical Imaging Conference (NSS/MIC); October 23-29, 2011; Valencia, Spain.

23. Lang N, Dawood M, Buther F, Schober O, Schafers M, Schafers K. Organ movement reduction in PET/CT using dual-gated list-mode acquisition. $\mathrm{Z} \mathrm{Med}$ Phys. 2006;16:93-100.

24. Martinez- Möller A, Zikic D, Botnar RM, et al. Dual cardiac-respiratory gated PET: implementation and results from a feasibility study. Eur J Nucl Med Mol Imaging. 2007;34:1447-1454.

25. Teräs M, Kokki T, Durand-Schaefer N, et al. Dual-gated cardiac PET: clinical feasibility study. Eur J Nucl Med Mol Imaging. 2010;37:505-516.

26. Koivumäki T, Nekolla SG, Furst S, et al. An integrated bioimpedance-ECG gating technique for respiratory and cardiac motion compensation in cardiac PET. Phys Med Biol. 2014;59:6373-6385.

27. Gigengack F, Ruthotto L, Burger M, Wolters CH, Jiang X, Schafers KP. Motion correction in dual gated cardiac PET using mass-preserving image registration. IEEE Trans Med Imaging. 2012;31:698-712.

28. Lamare F, Le Maitre A, Dawood M, et al. Evaluation of respiratory and cardiac motion correction schemes in dual gated PET/CT cardiac imaging. Med Phys. 2014;41:072504

29. Hong I, Jones J, Casey M. Elastic motion correction for cardiac pet studies. Paper presented at: Nuclear Science Symposium and Medical Imaging Conference (NSS/MIC); October 27 to November 2, 2013; Seoul, Korea.

30. Bousse A, Bertolli O, Atkinson D, et al. Maximum-likelihood joint image reconstruction/motion estimation in attenuation-corrected respiratory gated PET/ CT using a single attenuation map. IEEE Trans Med Imaging. August 3, 2015 [Epub ahead of print].

31. Slomka PJ, Le Meunier L, Hayes SW, et al. Comparison of myocardial perfusion 82Rb PET performed with CT- and transmission CT-based attenuation correction. J Nucl Med. 2008;49:1992-1998. 